\title{
Activités antitumorale et antimétastasique des alkylglycérols naturels : relation structure-activité
}

\author{
Anne-Laure DENIAU ${ }^{1}$ \\ Damien LE BOT ${ }^{1}$ \\ Paul MOSSET ${ }^{2}$ \\ Alain B. LEGRAND ${ }^{1}$ \\ 1 Laboratoire de pharmacologie moléculaire,
2 avenue du Pr Léon Bernard,
35043 Rennes cedex
<alain.legrand@univ-rennes1.fr>
2 École nationale supérieure de chimie de Rennes,
avenue du Général Leclerc,
CS 50837 Rennes cedex 7,
et université européenne de Bretagne
}

Les alkylglycérols (AKG) sont des éther-lipides présents dans la nature sous deux formes. La première est la forme du 1-0-alkyldialcyl-sn-glycérol. C'est cette forme qui est abondante dans les huiles de foie de certains requins. La seconde forme est celle des 1-0alkyl-sn-glycérophospholipides que l'on peut trouver dans différents tissus de l'organisme.

Les AKG sont notamment retrouvés dans les organes hématopoïétiques, la moelle osseuse, la rate, le foie, les leucocytes, également dans le lait. Ils sont présents en abondance dans le foie de certains requins et dans celui des chimères. Les AKG de I'huile de foie de requin constituent une famille où les espèces varient en fonction de la longueur et de l'insaturation de la chaîne. L'huile de foie de requin extraite de l'espèce Centrophorus squamosus contient 20 à $25 \%$ du poids de I'huile après écart du squalène. Cette huile est utilisée en médecine empirique dans les pays nordiques largement impliqués dans la pêche, comme les pays scandinaves ou le Japon, pour des propriétés cicatrisantes ou de stimulation des défenses immunitaires. Les AKG prédominants dans I'huile de foie de requin sont : I'alcool sélachylique (chaîne alkyle C18:1 n-9) qui représente à lui seul environ $60 \%$ du poids des AKG, I'alcool chimylique (C16:0) et l'alcool mono-insaturé correspondant (C16:1 n-7) représentant chacun de 10 à $15 \%$, et l'alcool batylique (C18:0) qui représente 2 à $5 \%$.

À partir de la seconde moitié du $x x^{\mathrm{e}}$ siècle, des études expérimentales ont cherché à

\begin{abstract}
Oral alkylglycerols (AKG) from shark liver oil (SLO) have multiple biological activities including immuno-stimulation and anti-tumour properties. In several cell types they are incorporated into phospholipids and therefore they may produce modified messengers and mediators following cellular activation of the phospholipase pathway. Structure of naturally occurring AKG vary depending on length and unsaturation of the alkyl chain. We synthesized six of the major natural constituants of AKG from SLO and tested their anti-tumour and anti-metastasis activities on a model of solid tumour grafted in mice. We found that unsaturated compounds were the most active for reducing tumour volume, lung metastasis number, and splenomegaly. Saturated compounds had weaker, no, or deleterious activities on this model. These data suggest that the selective use of unsaturated natural AKC would improve anti-tumour activities as compared to natural mix of AKG.
\end{abstract}

Key words: shark liver oil, bioactive lipids, ether lipids, alkylglycerols, anti-tumour activities

démontrer les activités biologiques de I'huile de foie de requin. Plusieurs activités ont été décrites, parmi lesquelles des activités hématopoiétiques, érythropoïétiques et thrombocytopoïétiques chez le rongeur. Chez des patientes traitées par radiothérapie pour un cancer du col de l'utérus, il fut observé une diminution de la thrombocytopénie induite par le traitement. Chez ces mêmes patientes, on observa également une réduction de la croissance tumorale et une diminution de la mortalité. Des effets antitumoraux ont par ailleurs été observés in vitro sur des cellules tumorales. De plus, des effets immunostimulants in vitro et in vivo ont été aussi démontrés.

Comment peut-on expliquer ces multiples activités sur le plan pharmacologique? On sait que les lipides servent de précurseurs à de nombreux messagers et médiateurs néoformés, qui jouent un rôle de premier plan dans les communications intra- et intercellulaires. Il existe de plus une plasticité dans la composition des lipides membranaires, qui dépend pour une part des apports externes. Notre hypothèse a été que les AKG pourraient s'intégrer dans les phospholipides membranaires pour donner des éther-phospholipides. Lors de l'activation d'un récepteur mettant en jeu la voie des phospholipases, ces étherphospholipides pourraient donner des seconds messagers et des médiateurs lipidiques modifiés structurellement par la fonction éther, et donc présentant des propriétés biologiques différentes.
Parmi les éther-lipides bioactifs qui pourraient apparaître, il y a le PAF (platelet-activating factor) dont la composition et la quantité pourraient être modifiées. Le 1-0-alkyl-2-acyl-sn-glycérol est un autre composé susceptible de modifier la signalisation cellulaire. C'est un analogue du diacylglycérol (DAG), le second messager activateur de la protéine-kinase C (PKC). Contrairement au DAG, cet analogue possède des activités inhibitrices de la PKC. D'autres éther-lipides naturels pourraient être amplifiés, par exemple l'acide 1-0-alkyllysophosphatidique qui est également un médiateur agissant sur des récepteurs.

Nous avons démontré quelques-unes de ces hypothèses sur des modèles in vitro. Les AKG tritiés peuvent s'incorporer dans des étherphospholipides de plusieurs types cellulaires comme les cellules endothéliales, les plaquettes ou les monocytes humains transformés THP1. Dans ces monocytes, les AKG participent à la production de PAF, et de plus, ils amplifient la production globale de PAF. Nous avons aussi démontré la production de 1-0-alkyl2-acyl-sn-glycérol par des cellules endothéliales cultivées en présence d'AKG, cette production est augmentée lors d'une stimulation.

Nous avons également étudié des effets immunostimulants de I'huile de foie de requin sur des truies en gestation et subissant une vaccination contre la maladie d'Aujeszki. L'huile de foie de requin s'oppose partiellement à l'anémie observée au cours de la gestation et amplifie la production d'immunoglobulines Aujeszki- 
spécifiques. Ces anticorps sont retrouvés à la fois dans le sérum et le colostrum. Les porcelets des truies traitées ont également des taux sériques d'anticorps spécifiques augmentés. Par ailleurs, les AKG passent dans le lait et induisent des effets de stimulation de l'hématopoïèse sur les cellules médiatrices de l'immunité. Globalement, tous ces effets aboutissent à un meilleur statut sanitaire et à une augmentation de la croissance des porcelets nés des truies traitées. Nous avons étudié les effets antitumoraux des AKG sur un modèle de souris recevant une greffe de tumeur solide (cellules 3LL). Sur ce modèle, nous avons étudié la croissance tumorale, le nombre et la taille des métastases, ainsi que la néoangiogenèse de la tumeur greffée. Les AKG aussi bien que I'huile de foie de requin réduisent significativement la croissance tumorale et le nombre de métastases pulmonaires. De plus, dans les tumeurs greffées, on observe une diminution d'un marqueur de l'endothélium : le facteur VIII (Von Willebrand), ce qui suggère une diminution de l'angiogenèse par les AKG. Sur les cellules 3LL cultivées, la cytotoxicité des AKG in vitro est faible, elle ne se manifeste à 72 heures que pour des concentrations égales ou supérieures à $100 \mu \mathrm{M}$.

La néoangiogenèse met en jeu des facteurs qui provoquent la prolifération des cellules endothéliales, tels que le VEGF ou le bFGF. Nous avons montré que les AKG à des concentrations égales ou supérieures à $20 \mu \mathrm{M}$ sont capables $d^{\prime}$ inhiber l'augmentation de prolifération des cellules endothéliales induite par le bFGF, suggérant là encore un possible effet inhibiteur sur la néoangiogenèse.

Pour évaluer l'activité de chacun des composés présents dans le mélange naturel, nous avons synthétisé six des principaux composés trouvés dans I'huile de foie de requin, quatre saturés à $12,14,16$ et 18 atomes de carbone et deux mono-insaturés : le C16:1 n-7 et le C18:1 n-9 en respectant l'asymétrie du carbone 2 du motif glycérol et la configuration cis des doubles liaisons des chaînes alkényles. Les effets de chacun de ces composés ont été évalués sur le volume tumoral à 20 jours de la greffe chez les souris. Les composés les plus actifs ont été les AKG C16:1 et C18:1, avec une faible activité pour l'AKG C12:0. Par contraste, I'AKG C18:0 tend à augmenter le volume tumoral de façon cependant non significative. Nous avons également évalué le nombre de métastases pulmonaires sous ces différents traitements et avons constaté, là encore, une très forte activité des composés C16:1 et C18:1 avec une activité moindre pour le C16:0 et, là encore, une tendance non significative à l'augmentation des métastases pulmonaires avec le C18:0. La greffe des tumeurs induit une augmentation très importante du poids de la rate. Le traitement avec les AKG insaturés $\mathrm{C} 16: 1$ et $\mathrm{C} 18: 1$ réduit fortement cette augmentation et ramène le poids de la rate à celui des rates de souris non traitées. Les AKG C14:0 et C16:0 ont un léger effet réducteur de ce poids. En revanche, I'AKG C18:0 a pour effet d'augmenter encore le poids de la rate par rapport à celui des souris greffées et non traitées. Ces effets contrastés sont en lien avec l'envahissement de la rate des souris greffées par des leucocytes immatures qui pourraient entraver la réponse immunologique antitumorale.

Pour évaluer une activité antiangiogénique des différents composés, nous avons testé leurs effets sur les cellules endothéliales de veines ombilicales humaines. La cytotoxicité pour tous ces composés apparaît de façon modérée à des concentrations supérieures ou égales à $25 \mu \mathrm{M}$. Les composés ayant une activité antiproliférative sur les cellules endothéliales à des concentrations inférieures à $25 \mu \mathrm{M}$ s'avèrent être les composés ayant une activité antitumorale : à savoir les AKG en C18:1, en C16:1 et en C16:0.

Parmi les mécanismes possibles permettant d'expliquer les différentes activités des AKG, on peut citer l'amplification de la production de PAF, lui-même impliqué dans les réponses immunologiques, et également la production du 1-0-alkyl-2-acyl-sn-glycérol cité plus haut. Son activité inhibitrice sur la PKC pourrait expliquer les effets antiprolifératifs et antiangiogéniques.

\section{Conclusion}

II apparaît que les AKG naturels insaturés 16:1 et 18:1 sont de loin les plus puissants antitumoraux et antimétastatiques, alors que le composé 18:0 (alcool batylique) est totalement dépourvu de ces activités et semble avoir un effet délétère notamment sur les réponses spléniques. Ces résultats soulignent l'intérêt qu'il y a, lorsqu'on recherche des effets antitumoraux, à utiliser les AKG insaturés isolés ou en mélange plutôt que l'ensemble des AKG naturels extraits de I'huile de foie de requin.

Remerciements. Ces études ont été réalisées avec I'aide de la Ligue nationale contre le cancer, de la région Bretagne et de la société Polaris (Pleuven, 29). 